\title{
PRECISION ASTROMETRY WITH ADAPTIVE OPTICS
}

\author{
P. B. Cameron, M. C. Britton, and S. R. Kulkarni \\ California Institute of Technology, Division Physics, Mathematics and Astronomy, MC 105-24, Pasadena, CA 91125, USA; pbc@astro.caltech.edu \\ Received 2008 May 13; accepted 2008 September 16; published 2008 December 10
}

\begin{abstract}
We investigate the limits of ground-based astrometry with adaptive optics using the core of the Galactic globular cluster M5. Adaptive optics systems provide near diffraction-limit imaging with the world's largest telescopes. The substantial improvement in both resolution and signal-to-noise ratio enables high-precision astrometry from the ground. We describe the dominant systematic errors that typically limit ground-based differential astrometry, and enumerate observational considerations for mitigating their effects. After implementing these measures, we find that the dominant limitation on astrometric performance in this experiment is caused by tilt anisoplanatism. We then present an optimal estimation technique for measuring the position of one star relative to a grid of reference stars in the face of this correlated random noise source. Our methodology has the advantage of reducing the astrometric errors to $\sim 1 / \sqrt{t}$ and faster than the square root of the number of reference stars, effectively eliminating noise caused by atmospheric tilt to the point that astrometric performance is limited by centering accuracy. Using 50 reference stars, we demonstrate a single-epoch astrometric precision of $\approx 1$ mas in $1 \mathrm{~s}$, decreasing to $\lesssim 100 \mu$ as in 2 minutes of integration time at the Hale 200 inch telescope. We also show that our astrometry is accurate to $\lesssim 100 \mu$ as for observations separated by 2 months. Finally, we discuss the limits and potential of differential astrometry with current and next-generation large-aperture telescopes. At this level of accuracy, numerous astrometric applications become accessible, including planet detection, astrometric microlensing signatures, and kinematics of distant Galactic stellar populations.
\end{abstract}

Key words: astrometry - globular clusters: individual (M5) - instrumentation: adaptive optics - methods: statistical

\section{INTRODUCTION}

The benefits of astrometry have long been clear to astronomers. Measurements of parallax and proper motion yield model-independent determinations of fundamental quantities like distance and velocity. It is not surprising that astrometry has motivated a wide variety of observational programs using many different techniques to answer fundamental questions in astrophysics. Potential applications span a wide range of physical scales including planet detection, reconstruction of the Milky Way's formation, and tests of $\Lambda$ cold dark matter (CDM) cosmology (e.g., Unwin et al. 2008).

The most ubiquitous astrometric measurements have been carried out with ground-based telescopes in the seeing limit. Monet et al. (1992) conducted visible light measurements of 72 stars $(V=15-20)$ using the $1.55 \mathrm{~m}$ US Naval Observatory astrometric reflector. This program achieved a singleepoch measurement precision of $\approx 4$ milliarcseconds (mas), and parallax accuracies ranging from 0.5 to 3 mas over $\sim$ 5 yr baselines. Pravdo \& Shaklan (1996) performed visible light measurements of stars in the cluster NGC 2420 $(V=13-16)$ and achieved a single-epoch precision of $\approx 150 \mu$ as in $1 \mathrm{hr}$, which motivated an astrometric survey for low-mass companions to nearby stars (e.g., Pravdo et al. 2004). More recently, 200-300 $\mu$ as astrometric precision has been demonstrated with the Very Large Telescope (VLT)/FORS in the visible (Lazorenko 2006; Lazorenko et al. 2007). Each of the above programs employed relatively narrowfield visible imagers (a few square arcminutes) to perform differential astrometry; however, the increasing availability of wide angle imagers has motivated studies over larger fields. Anderson et al. (2006) performed similar experiments using a $33^{\prime} \times 34^{\prime}$ visible camera on the European Southern Observatory (ESO) $2.2 \mathrm{~m}$ telescope, which resulted in 7 mas single-epoch precision.
Ground-based interferometers provide an alternative method for performing high-precision astrometry, typically over very narrow fields relative to a single reference star. The Palomar Testbed Interferometer has used phase-referencing to achieve astrometric accuracies $\approx 100 \mu$ as for a $30^{\prime \prime}$ binary (Lane et al. 2000 ) and $\approx 20 \mu$ as over years for binaries with separations $\lesssim 1^{\prime \prime}$ (Muterspaugh et al. 2006). Due to its $40 \mathrm{~cm}$ apertures, this instrument is limited to targets with $K_{s}<6$. Large aperture, ground-based interferometers equipped with adaptive optics systems, such as those at Keck (Colavita \& Wizinowich 2003) and the VLT (Glindemann et al. 2000), can perform at levels similar to fainter limiting magnitudes (e.g., Boden et al. 2007).

Differential astrometric accuracies achieved in both single aperture and interferometric ground-based programs are fundamentally limited by atmospheric effects. In the seeing limit, single aperture observations suffer from image quality degradation and interferometers lose visibility fringe coherence due to atmospheric turbulence. In addition, all groundbased single aperture programs suffer from systematic effects due to differential atmospheric refraction and optical distortions.

Space-based observatories are one possible method for avoiding the effects of atmospheric turbulence. Hipparcos was the first space-based mission with primarily astrometric goals, and achieved $\lesssim 1$ mas astrometry over the mission lifetime on bright targets ( $V \lesssim 9$ mag; Perryman et al. 1997). Currently, the only space-based telescope that can perform high-precision astrometry is Hubble. Both the imagers and Fine Guidance Sensor (FGS) have been characterized and well utilized for astrometry at the $\lesssim 1$ mas level (e.g., Anderson \& King 2000, 2003b; Benedict et al. 2003). Two complimentary future space missions are aimed at achieving levels of astrometric performance 2-3 orders of magnitude below the Hubble performance levels. GAIA 
will catalog roughly one billion stars to $V \approx 20$ mag over the entire sky with parallax accuracies ranging from 10 to $300 \mu$ as depending on the magnitude (Perryman et al. 2001). The Space Interferometry Mission (SIM) will take a pointed approach, and enable microarcsecond astrometry on Galactic and extragalactic targets (Unwin et al. 2008).

Ground-based adaptive optics (AO) offer an alternative, more easily accessible, and cost-effective method for overcoming atmospheric turbulence over small fields ( $\lesssim$ arcminute). The current generation of astronomical AO systems provide diffraction-limited image quality at near-infrared (NIR) wavelengths. Achieving the telescope's diffraction limit and the resulting boost in the signal-to-noise ratio $(\mathrm{S} / \mathrm{N})$ prove to be a powerful combination for astrometry. These two effects reduce the errors in determining stellar centers, increase the number of possible reference stars at small separations, and allow techniques for mitigating systematics (e.g., use of narrowband filters to eliminate chromatic refraction; see Section 2).

The marked improvement in wavefront sensor technology and the development of laser beacons have rapidly increased the usable sky coverage of these systems (e.g., Wizinowich et al. 2006). The increase in sky coverage, operation in the NIR, gain in the $\mathrm{S} / \mathrm{N}$, and the diffraction-limited image quality make astrometry with AO amenable to numerous Galactic applications spanning a wide number of fields: detection of astrometric companions, the improved determination of the mass-luminosity relation of stars, and the formation and evolution of compact objects (Unwin et al. 2008).

Here we present an optimal estimation technique, appropriate for mitigating the astrometric errors arising in $\mathrm{AO}$ observations, and demonstrate its potential with multiepoch imaging of the core of the globular cluster M5 using the Hale 200 inch telescope. We are able to achieve $\lesssim 100 \mu$ as astrometric precision in 2 minutes, and have maintained this accuracy over 2 months. In Section 2, we discuss the dominant noise terms that arise in ground-based astrometry and the experimental techniques we have adopted to control them. We describe the framework of our reduction model and illustrate its salient properties with a numerical simulation in Section 4. We describe the observations of M5 and the results of applying the optimal estimation technique to the data in Sections 3 and 5. This is followed in Section 6 by a discussion of the role and potential of $\mathrm{AO}$ in ground-based astrometry with current and future large aperture telescopes.

\section{ASTROMETRIC ERROR TERMS IN GROUND-BASED ASTROMETRY}

Ground-based optical and IR imaging observations suffer from a number of errors that limit the accuracy and precision of astrometric measurements. Relative to seeing-limited observations, the diffraction-limited image quality afforded by AO modifies the relative importance of these error terms. This section describes the four largest effects, and indicates observational considerations utilized in this experiment aimed at mitigating them.

\subsection{Differential Tilt Jitter}

With AO, the image motion of the guide star is removed with a flat tip-tilt mirror. This stabilizes the image of the guide star with respect to the imager to high accuracy. Any residual tip-tilt error is removed in subsequent analysis by calculating only differential offsets between the target of astrometry (not necessarily the AO guide star) and the reference stars. However, the difference in the tilt component of turbulence along any two lines of sight (LOS) in the field of view (FOV) causes a correlated, stochastic change in their measured separation, known as differential atmospheric tilt jitter.

More specifically, in propagating through the atmosphere to reach the telescope aperture, light from the target star and light from a reference star at a finite angular offset traverse different columns of atmospheric turbulence that are sheared. Differential atmospheric tilt jitter arises from the decorrelation in the tilt component of the wavefront phase aberration arising from this shearing effect. This differential tilt leads to a random, achromatic, and anisotropic fluctuation in the relative displacement of the two objects. The three-term approximation to the parallel and perpendicular components of the variance arising from differential atmospheric tilt jitter, assuming Kolmogorov turbulence, is given by (Sasiela 1994)

$$
\begin{aligned}
{\left[\begin{array}{c}
\sigma_{\|, \mathrm{TJ}}^{2} \\
\sigma_{\perp, \mathrm{TJ}}^{2}
\end{array}\right]=} & 2.67 \frac{\mu_{2}}{D^{1 / 3}}\left(\frac{\theta}{D}\right)^{2}\left[\begin{array}{l}
3 \\
1
\end{array}\right]-3.68 \frac{\mu_{4}}{D^{1 / 3}}\left(\frac{\theta}{D}\right)^{4}\left[\begin{array}{l}
5 \\
1
\end{array}\right] \\
& +2.35 \frac{\mu_{14 / 3}}{D^{1 / 3}}\left(\frac{\theta}{D}\right)^{14 / 3}\left[\begin{array}{c}
17 / 3 \\
1
\end{array}\right] .
\end{aligned}
$$

In this equation, $D$ is the telescope diameter and $\theta$ is the angular separation of the stars. The turbulence moments $\mu_{m}$ are defined as

$$
\mu_{m}=\sec ^{m+1} \xi \int_{0}^{\infty} d h C_{n}^{2}(h) h^{m},
$$

where $h$ is the altitude, $\xi$ is the zenith angle, and $C_{n}^{2}(h)$ is the vertical strength of atmospheric turbulence. Typical $C_{n}^{2}(h)$ profiles yield $\sigma_{\|, \mathrm{TJ}} \approx 20-30$ mas for a $20^{\prime \prime}$ binary when observed with a $5 \mathrm{~m}$ aperture. Note that the variance from differential tilt is a random error, and, thus, is also $\propto \tau_{\mathrm{TJ}} / t$, where $\tau_{\mathrm{TJ}}$ is the tilt jitter timescale (on the order of the wind crossing time over the aperture; see Section 5) and $t$ is the integration time.

\subsection{Distortion}

The largest instrumental systematic that limits the accuracy of astrometry in any optical system is geometric distortion. These distortions can be stable-resulting from unavoidable errors in the shape or placement of optics_-or dynamic_-resulting from the flexure or replacement of optics.

If geometric distortions are stable, then a number of strategies can be employed to mitigate their effect. One method is to model the distortion to high accuracy; the most notable example is the calibration of the Hubble Space Telescope (HST; e.g., Anderson \& King 2003a). This is particularly important for data sets obtained with multiple instruments or those that use the technique of dithering, since knowledge of the distortion is necessary to place stellar positions in a globally-correct reference frame. Alternatively, one could use a consistent optical prescription from epoch to epoch by using the same instrument and placing the field at the same location and orientation on the detector. Here we use both a distortion solution and a single, consistent dither position to achieve accurate astrometry.

Any changes in the geometric distortion must be tracked through routine, consistent calibration. The question of stability is particularly important at the Hale 200 inch telescope, since the AO system and the imaging camera (Palomar Adaptive Optics System (PALAO) and Palomar High Angular Resolution Observer (PHARO), respectively; see Section 3 ) are mounted at 
the Cassegrain focus, and PHARO undergoes a few warming/ cooling cycles per month (see Section 3 ). The PHARO distortion solution $^{1}$ by Metchev (2006) accounts for changes in the orientation of the telescope (which are relatively small for our experimental design), but the overall stability of the system is best verified with on-sky data. One of the purposes of the data presented here is to track the system stability. We find that the combination of the Hale Telescope, PALAO, and PHARO is capable of delivering $\lesssim 100 \mu$ as astrometry.

\subsection{Atmospheric Refraction}

Refraction by the Earth's atmosphere causes an angular deflection of light from a star, resulting in an apparent change in its position. The magnitude of this deflection depends on the wavelength and the atmospheric column depth encountered by an incoming ray. The former effect is chromatic, while the latter is achromatic. The error induced by differential chromatic refraction (DCR) has proven to be an important, and sometimes the dominant, astrometric limitation in ground-based efforts (e.g., Monet et al. 1992; Pravdo \& Shaklan 1996; Anderson et al. 2006; Lazorenko 2006). These studies have shown that DCR can contribute $\approx 0.1-1$ mas of error depending on the wavelength and strategy of the observations.

The observations presented here were conducted using a $\mathrm{Br}-\gamma$ filter at $2.166 \mu \mathrm{m}$ with a narrow bandpass of $0.02 \mu \mathrm{m}$ to suppress $\mathrm{DCR}$. The increased $\mathrm{S} / \mathrm{N}$ provided by $\mathrm{AO}$ allows sufficient reference stars to be detected even through such a narrow filter in a short exposure time. We reach $K_{s} \approx 15 \mathrm{mag}$ in our $1.4 \mathrm{~s}$ exposures through this filter with the Hale 200 inch telescope (see Section 3). In addition, observations were acquired over a relatively narrow range of airmass $(1.17-1.27)$ at each epoch to minimize the achromatic differential refraction.

In order to estimate the effect of atmospheric refraction on our data, we took the asterism in the core of M5 and refracted it to 37 and $32^{\circ}$ elevation with the parallactic angles appropriate for the observations on 2007 May 28 using the slarefro function distributed with the STARLINK library (Gubler \& Tytler 1998). The root mean square (rms) deviation in reference star positions between these two zenith angles was $\lesssim 250 \mu$ as and the shift in the guide star position with respect to the grid (see Section 4) was $\approx 10 \mu$ as. Thus, our consistent zenith angle of observations, narrowband filter, and observations in the NIR (where the refraction is more benign) make the contribution of this effect negligible for our purposes, and we make no effort to correct for it.

Performing a similar experiment using a broadband $K$ filter with a field of $\approx 5000 \mathrm{~K}$ reference stars and $\mathrm{a} \approx 3000 \mathrm{~K}$ target would lead to a systematic shift of $\approx 100 \mu$ as between zenith angles separated by $10^{\circ}$, which would be detectable by this experiment. Consequently, for observations where broadband filters are necessary, refraction effects must be considered and corrected.

\subsection{Measurement Noise}

In the case of a perfect optical system, a perfect detector, and no atmosphere, the astrometric precision is limited to one's ability to calculate stellar centers. The centering precision is determined by measurement noise, and we will use the two terms

\footnotetext{
See also http://www.astro.ucla.edu/ metchev/ao.html
}

interchangeably. For a monopupil telescope, the uncertainty is

$$
\sigma_{\text {meas }}=\frac{\lambda}{\pi D} \frac{1}{\mathrm{SNR}}=284 \mu \mathrm{as}\left(\frac{\lambda}{2.17 \mu \mathrm{m}}\right)\left(\frac{5 \mathrm{~m}}{D}\right)\left(\frac{100}{\mathrm{SNR}}\right)
$$

(Lindegren 1978). AO allow us to achieve the diffraction limit even in the presence of the atmosphere and substantially boosts the SNR over the seeing-limited case, thereby decreasing measurement noise and improving astrometric precision.

In practice, the centering of a given stellar image is limited by spatial and temporal variations in the $\mathrm{AO}$ point-spread function (PSF). A great deal of time and effort has been spent determining the AO PSF and producing software packages to perform PSF fitting (e.g., Diolaiti et al. 2000; Britton 2006). However, any PSF-fitting software package is capable of calculating image positions at $\mathrm{a} \lesssim 0.01$ pixel level in a single image. For the observations considered here, this is $\lesssim 2$ mas, a factor of 5-10 larger than the measurement noise in Equation (3), but this is much smaller than the tilt jitter mentioned in Section 2.1. As such, we have chosen to use simple and widely-available PSF centering software (DAOPHOT; Stetson 1987; see Section 3).

\section{OBSERVATIONS AND DATA REDUCTION}

We observed the globular cluster M5 on three dates spanning 2 months (see Table 1 for a summary of observations) using the Hale 200 inch telescope and the PHARO (Hayward et al. 2001) assisted by the PALAO (Troy et al. 2000). The globular cluster M5 was chosen for its relatively large distance of $\approx 7.5 \mathrm{kpc}$ from the Sun, low velocity dispersion of $\approx 5 \mathrm{~km} \mathrm{~s}^{-1}$, and the availability of guide stars near the cluster core (Pryor \& Meylan 1993; Harris 1996). This combination of distance and velocity

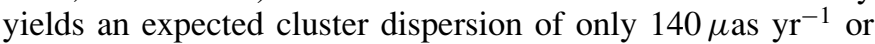
$20 \mu$ as over our 2 month observing span. We acquired 400-600 images per night. A typical image can be found in Figure 1. The guide star is a red giant branch (RGB) member of the globular cluster with $V \approx 12.6$ mag (Sandquist \& Bolte 2004). The cluster was imaged through the narrowband $\mathrm{Br}-\gamma$ filter (central wavelength is $2.166 \mu \mathrm{m}$ and bandpass is $0.02 \mu \mathrm{m}$ ) using the $25^{\prime \prime} \times 25^{\prime \prime}$ narrow-field channel $\left(0^{\prime \prime} .025\right.$ pixel $\left.^{-1}\right)$, which oversamples the 87 mas diffraction-limited PSF. The brightest star filled the detector to $10 \%$ of the maximum well-depth in the $1.4 \mathrm{~s}$ exposure time, well within the linear regime of the detector.

Contemporaneous measurements of the atmospheric turbulence profile were acquired with a differential image motion monitor (DIMM) and multiaperture scintillation sensor (MASS), which have been deployed as a single unit in a dome at Palomar Observatory (Thomsen et al. 2007; Kornilov et al. 2007). These turbulence profile measurements permitted an independent estimate of the magnitude of differential tilt jitter (computed using Equation 1).

We processed the raw images by subtracting dark frames and removing bad pixels from the analysis. Flat-field calibration was performed using twilight sky flats. Sky subtraction was accomplished by forming the median of the dithered frames taken outside of the cluster and subtracting this median from each exposure. The photometry and astrometry of each star were extracted using PSF fitting as implemented by the DAOPHOT package in PyRAF. ${ }^{2}$ DAOPHOT is not optimized for astrometry (see, e.g., Anderson \& King 2000), and since our measurement

\footnotetext{
2 PyRAF is a product of Space Telescope Science Institute, which is operated
} by AURA, Inc. for NASA. 

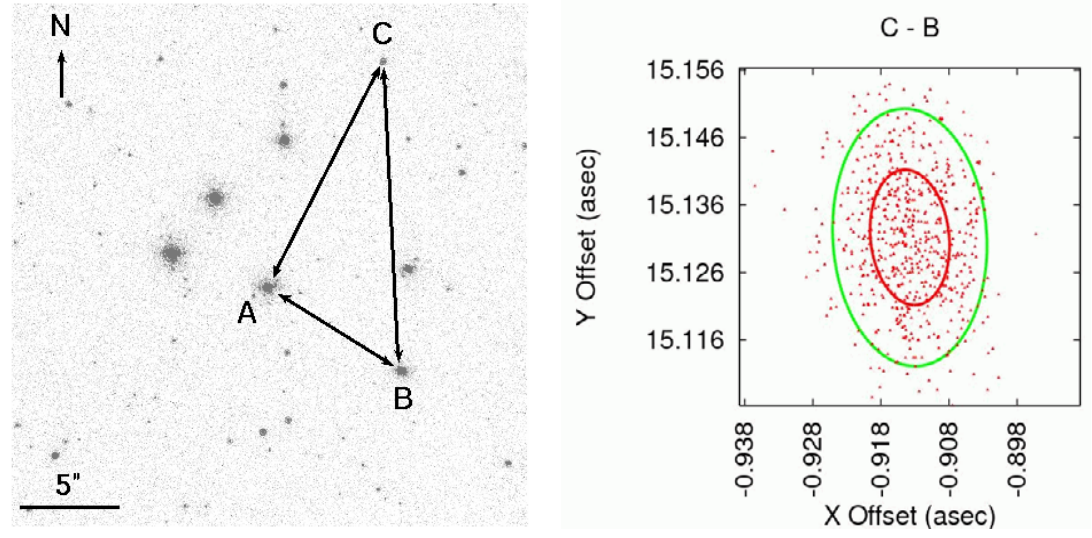

C-A
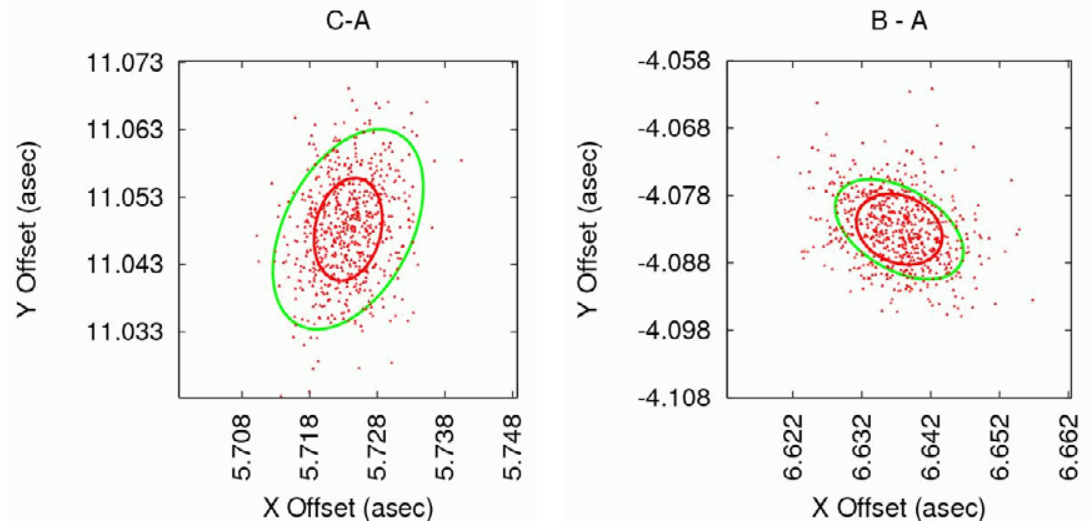

Figure 1. Top left: image of the core of the globular cluster M5 in $1.4 \mathrm{~s}$ through the narrowband Br- $\gamma$ filter. The AO guide star is labeled as star "A," and is one of 82 detected stars in the image. The additional plots show the measured $x-y$ angular separation of each pair of stars denoted by the arrows in 600 images taken on 2007 May 28. These plots show the clear signature of anisotropic differential atmospheric tilt jitter as predicted from Equation (1). The measured (red) and predicted (green) $1 \sigma$ error ellipses are overplotted. We see that temporal averaging over the $1.4 \mathrm{~s}$ exposure time has reduced the measured variance with respect to that predicted from the DIMM/MASS measurements and Equation (1).

Table 1

Observations

\begin{tabular}{lcccccccc}
\hline \hline Date & Time & $\begin{array}{c}\text { Integration Time } \\
(\mathrm{sec})\end{array}$ & Airmass & $\begin{array}{c}\text { Seeing } \\
(\mathrm{asecs})\end{array}$ & $\begin{array}{c}\theta_{0}{ }^{\mathrm{a}} \\
(\mathrm{asecs})\end{array}$ & $\begin{array}{c}\mu_{2} \\
\left(\mathrm{~m}^{7 / 3}\right)\end{array}$ & $\begin{array}{c}\mu_{4} \\
\left(\mathrm{~m}^{13 / 3}\right)\end{array}$ & $\begin{array}{c}\mu_{14 / 3} \\
\left(\mathrm{~m}^{15 / 3}\right)\end{array}$ \\
\hline $2007-05-28$ & $05: 18: 29-06: 19: 29$ & 890 & $1.26-1.18$ & 1.22 & 2.34 & $1.01 \mathrm{e}-5$ & $3.82 \mathrm{e} 3$ & $2.77 \mathrm{e} 6$ \\
$2007-05-29$ & $05: 58: 26-06: 48: 30$ & 570 & $1.19-1.17$ & 1.39 & 2.16 & $1.14 \mathrm{e}-5$ & $3.89 \mathrm{e} 3$ & $2.82 \mathrm{e} 6$ \\
$2007-07-22$ & $03: 57: 12-04: 40: 36$ & 630 & $1.20-1.27$ & 1.05 & 1.66 & $1.74 \mathrm{e}-5$ & $6.46 \mathrm{e} 3$ & $4.71 \mathrm{e} 6$ \\
\hline
\end{tabular}

Note. ${ }^{a}$ Calculated at a wavelength of $0.5 \mu \mathrm{m}$. These quantities scale as $\lambda^{1 / 5}$ and $\lambda^{6 / 5}$, respectively.

model reduces the noise due to atmospheric turbulence, our single-epoch precision could be improved with a more careful centering technique (see Sections 4.4 and 5.2). However, our astrometric accuracy over 2 months is not limited by this choice (see Section 5.3). We used the four brightest stars in the field to derive a model PSF that is assumed to be constant over the field, and calibrated the image zeropoints using Two Micron All Sky Survey (2MASS) and find that the guide star has $K_{s} \approx 9.1 \mathrm{mag}$.

\section{GRID ASTROMETRY FOR GROUND-BASED AO OBSERVATIONS}

After controlling for distortion and atmospheric refraction, the dominant astrometric errors are caused by differential atmospheric tilt jitter and measurement noise. In this section, we present a general framework for measuring the position of a star relative to a grid of reference stars in the face of these noise sources. This framework has two key ingredients. The first is the covariance matrix $\left(\boldsymbol{\Sigma}_{\mathbf{d}}\right)$, which encapsulates the relevant statistical uncertainties for astrometry with $\mathrm{AO}$. The second is the weight matrix $(\mathbf{W})$, which determines how the differential measurements between the target star and the reference stars are combined to calculate the target's position relative to the grid.

\subsection{Measurement Model}

The fundamental quantity in differential astrometry is the measured angular offset between a pair of stars. We will denote the angular distance between two stars, $i$ and $j$, as $\vec{d}_{i j}$. Since $\vec{d}_{i j}$ is measured from an image, we will denote its components in the Cartesian coordinate system of the detector, simply

$$
\vec{d}_{i j}=\left[\begin{array}{l}
x_{j}-x_{i} \\
y_{j}-y_{i}
\end{array}\right] \equiv\left[\begin{array}{l}
x_{i j} \\
y_{i j}
\end{array}\right],
$$

where we have introduced the notation $x_{i j} \equiv x_{j}-x_{i}$ and likewise for $y$. The variance in the angular separation between two stars 
is given by

$$
\left[\begin{array}{l}
\sigma_{\|}^{2} \\
\sigma_{\perp}^{2}
\end{array}\right]=\left[\begin{array}{l}
\sigma_{\|, \text {meas }}^{2} \\
\sigma_{\perp, \text { meas }}^{2}
\end{array}\right]+\frac{\tau_{\mathrm{TJ}}}{t}\left[\begin{array}{l}
\sigma_{\|, \mathrm{TJ}}^{2} \\
\sigma_{\perp, \mathrm{TJ}}^{2}
\end{array}\right]
$$

where $\sigma_{\| \text {meas }}^{2}$ is the sum of the squares of the centering errors of each star parallel to the axis connecting the pair (and similarly for the perpendicular variance), and the remaining terms are as defined in Section 2.1.

Measurement of the offset between the target star (which we will denote with a subscript $i=0$ ) and each of the $N$ reference stars results in a set of $N$ vectors, $\vec{d}_{0 i}$. For simplicity, we will write these measured offsets as a single-column vector,

$$
\mathbf{d}=\left[x_{01}, \ldots, x_{0 N}, y_{01}, \ldots, y_{0 N}\right]^{\mathrm{T}} .
$$

The goal of differential astrometry is to use $\mathbf{d}$ to determine the position of the target star with respect to the reference grid of stars at each epoch.

There are many possible ways to construct the position of the astrometric target from a given d. Here we use the most general linear combination of the angular offsets, namely

$$
\vec{p}=\mathbf{W d},
$$

where $\mathbf{W}$ is the $2 \times 2 N$ weight matrix, given by

$$
\mathbf{W}=\left[\begin{array}{llllll}
w_{x x, 01} & \cdots & w_{x x, 0 N} & w_{x y, 01} & \cdots & w_{x y, 0 N} \\
w_{y x, 01} & \cdots & w_{y x, 0 N} & w_{y y, 01} & \cdots & w_{y y, 0 N}
\end{array}\right]
$$

We have used the notation $w_{x y, 0 i}$ to denote the weighting of the offset from the target star to star $i$ in the $y$-direction, used to determine the $x$ component of the target's position, $\vec{p}$. For example, for a standard average of the $x$ and $y$ measurements to calculate $\vec{p}$, we would assign all the $w_{x x, 0 i}=w_{y y, 0 i}=1 / N$ and $w_{x y, 0 i}=w_{y x, 0 i}=0$.

In principle, we are free to assign weights in any manner we please. However, we find it convenient to choose the weights such that they satisfy

$$
\begin{aligned}
\sum_{i} w_{x x, 0 i} & =1, & \sum_{i} w_{y y, 0 i} & =1, \\
\sum_{i} w_{x y, 0 i} & =0, & \sum_{i} w_{y x, 0 i} & =0 .
\end{aligned}
$$

These constraints ensure that the components of $\vec{p}$ have physical units (e.g., pixels or arcseconds) and that its components are measured in the same coordinate system as d (presumably the detector coordinates). As a consequence, $\vec{p}$ represents the position of the target star in the sense that a proper motion of the target, $\vec{\epsilon}$, with respect to the fixed grid between two epochs will cause a change, $\vec{p} \rightarrow \vec{p}+\vec{\epsilon}$.

In order to determine if any change in $\vec{p}$ over time is meaningful, we must understand its statistical properties. Both differential tilt jitter and measurement errors are assumed to follow Gaussian statistics, so that each instance of target-reference grid offset measurements, $\mathbf{d}$, is drawn from a multivariate normal probability distribution:

$$
P(\mathbf{d})=\frac{1}{\sqrt{2 \pi \operatorname{det} \Sigma_{\mathbf{d}}}} \exp \left(\frac{1}{2}[\mathbf{d}-\overline{\mathbf{d}}]^{\mathrm{T}} \boldsymbol{\Sigma}_{\mathbf{d}}^{-1}[\mathbf{d}-\overline{\mathbf{d}}]\right),
$$

where $\boldsymbol{\Sigma}_{\mathbf{d}}$ is the covariance matrix and the bars above symbols denote using the average value of each matrix entry.
The statistics of $\vec{p}$ follow in a straightforward manner from Equation (10), given our choice in Equation (7). Since $\vec{p}$ is a linear function of $\mathbf{d}$, each $\vec{p}$ is also drawn from a multivariate normal probability distribution with the covariance matrix

$$
\boldsymbol{\Sigma}_{\mathbf{p}}=\mathbf{W}^{\mathrm{T}} \boldsymbol{\Sigma}_{\mathbf{d}} \mathbf{W},
$$

and the uncertainties of $\vec{p}$ are described by the eigenvectors and eigenvalues of $\boldsymbol{\Sigma}_{\mathbf{p}}$. Thus, our goal of optimally determining the target's position requires calculating the covariance matrix, $\boldsymbol{\Sigma}_{\mathbf{d}}$, from data or theory, and choosing $\mathbf{W}$ to minimize the eigenvalues of $\boldsymbol{\Sigma}_{\mathbf{p}}$.

\subsection{The Covariance Matrix}

We have chosen to measure positions and offsets in the Cartesian coordinates of the detector, so the form of the covariance matrix, given our above definitions, is

$$
\Sigma_{\mathbf{d}}=\left(\begin{array}{cccccc}
\left\langle\left(\Delta x_{01}\right)^{2}\right\rangle & \cdots & \left\langle\left(\Delta x_{01}\right)\left(\Delta x_{0 N}\right)\right\rangle & \left\langle\left(\Delta x_{01}\right)\left(\Delta y_{01}\right)\right\rangle & \cdots & \left\langle\left(\Delta x_{01}\right)\left(\Delta y_{0 N}\right)\right\rangle \\
& \ddots & \vdots & \vdots & \ddots & \vdots \\
& & \left\langle\left(\Delta x_{0 N}\right)^{2}\right\rangle & \left\langle\left(\Delta x_{0 N}\right)\left(\Delta y_{01}\right)\right\rangle & \cdots & \left\langle\left(\Delta x_{0 N}\right)\left(\Delta y_{0 N}\right)\right\rangle \\
& & & \left\langle\left(\Delta y_{01}\right)^{2}\right\rangle & \cdots & \left\langle\left(\Delta y_{01}\right)\left(\Delta y_{0 N}\right)\right\rangle \\
& & & & \ddots & \vdots \\
\text { symmetric } & & & & & \left\langle\left(\Delta y_{0 N}\right)^{2}\right\rangle
\end{array}\right),
$$

where we have written as $\Delta x_{i j} \equiv\left(x_{i j}-\bar{x}_{i j}\right)$ to simplify the notation (likewise for $y$ ).

The total covariance matrix has contributions from centering errors and differential atmospheric tilt jitter. Since these contributions are independent, the total covariance matrix can be written as $\boldsymbol{\Sigma}_{\mathbf{d}}=\boldsymbol{\Sigma}_{\text {meas }}+\boldsymbol{\Sigma}_{\mathrm{TJ}}$, and each term can be derived separately.

\subsubsection{The Covariance Matrix for Measurement Noise}

In the absence of differential tilt jitter, it is straightforward to construct the covariance matrix for measurement noise alone, $\Sigma_{\text {meas. }}$. The diagonal terms can be written as

$$
\left\langle\Delta x_{0 i}^{2}\right\rangle \equiv \sigma_{x, 0 i}^{2}=\sigma_{x, 0}^{2}+\sigma_{x, i}^{2},
$$

where $\sigma_{x, i}$ and $\sigma_{x, 0}$ are the uncertainties in determining the $x$-position of star $i$ and the target star, respectively. For the offdiagonal terms $\left\langle\Delta x_{0 i} \Delta x_{0 j}\right\rangle$, we can use the fact that

$$
\begin{aligned}
\left\langle\Delta x_{0 i} \Delta x_{0 j}\right\rangle & =\frac{1}{2}\left\langle\left\{\Delta x_{0 i}^{2}+\Delta x_{0 j}^{2}-\left[\Delta x_{0 i}-\Delta x_{0 j}\right]^{2}\right\}\right\rangle \\
& =\frac{1}{2}\left\{\left\langle\Delta x_{0 i}^{2}\right\rangle+\left\langle\Delta x_{0 j}^{2}\right\rangle-\left\langle\left[\Delta x_{0 i}-\Delta x_{0 j}\right]^{2}\right\rangle\right\} \\
& =\frac{1}{2}\left\{\left\langle\Delta x_{0 i}^{2}\right\rangle+\left\langle\Delta x_{0 j}^{2}\right\rangle-\left\langle\Delta x_{i j}^{2}\right\rangle\right\} \\
& =\frac{1}{2}\left(\sigma_{x, 0 i}^{2}+\sigma_{x, 0 j}^{2}-\sigma_{x, i j}^{2}\right) \\
& =\sigma_{x, 0}^{2}
\end{aligned}
$$

where we have used only algebra and the above definitions. Equation (14) is the obvious result of the fact that the measurements of the target star's coordinates are common to all differential measurements, and so its uncertainty appears in all the offdiagonal covariance terms, $\left\langle\Delta x_{0 i} \Delta x_{0 j}\right\rangle$ and $\left\langle\Delta y_{0 i} \Delta y_{0 j}\right\rangle$. However, the cross-terms involving both $x$ and $y$ (e.g., $\left.\left\langle\Delta x_{0 i} \Delta y_{0 j}\right\rangle\right)$ vanish because $\sigma_{x, 0}$ and $\sigma_{y, 0}$ are uncorrelated for measurement noise alone. 


\subsubsection{The Covariance Matrix for Differential Tilt Jitter}

The covariance matrix for differential atmospheric tilt jitter between a pair of stars is diagonal when written in an orthogonal coordinate system with one axis lying along the separation axis of the binary. From Equation (1), we see that it can be written as

$$
\begin{aligned}
\Sigma_{\text {pair }} & =\left(\begin{array}{cc}
\left\langle\left(d_{\|}-\bar{d}_{\|}\right)^{2}\right\rangle & \left\langle\left(d_{\|}-\bar{d}_{\|}\right)\left(d_{\perp}-\bar{d}_{\perp}\right)\right\rangle \\
\left\langle\left(d_{\|}-\bar{d}_{\|}\right)\left(d_{\perp}-\bar{d}_{\perp}\right)\right\rangle & \left\langle\left(d_{\perp}-\bar{d}_{\perp}\right)^{2}\right\rangle
\end{array}\right) \\
& =\left(\begin{array}{cc}
\sigma_{\|, \mathrm{TJ}}^{2} & 0 \\
0 & \sigma_{\perp, \mathrm{TJ}}^{2}
\end{array}\right),
\end{aligned}
$$

where $d_{\|}$and $d_{\perp}$ are the angular offsets parallel and perpendicular to the axis connecting the pair of stars, respectively.

For a general field of $N$ stars, no coordinate system exists that diagonalizes the full tilt jitter covariance matrix, $\boldsymbol{\Sigma}_{\mathrm{TJ}}$. But we can begin computing the entries by rotating $\boldsymbol{\Sigma}_{\text {pair }}$ into our $x-y$ coordinates via $\mathbf{R}^{\mathrm{T}} \boldsymbol{\Sigma}_{\text {pair }} \mathbf{R}$, where

$$
\mathbf{R}=\left(\begin{array}{cc}
\cos \phi & \sin \phi \\
-\sin \phi & \cos \phi
\end{array}\right)
$$

The result is

$\mathbf{R}^{\mathrm{T}} \boldsymbol{\Sigma}_{\text {pair }} \mathbf{R}=$

$\left(\begin{array}{ll}\sigma_{\|, 0 i}^{2} \cos ^{2} \phi_{0 i}+\sigma_{\perp, 0 i}^{2} \sin ^{2} \phi_{0 i} & \left(\sigma_{\|, 0 i}^{2}-\sigma_{\perp, 0 i}^{2}\right) \cos \phi_{0 i} \sin \phi_{0 i} \\ \left(\sigma_{\|, 0 i}^{2}-\sigma_{\perp, 0 i}^{2}\right) \cos \phi_{0 i} \sin \phi_{0 i} & \sigma_{\|, 0 i}^{2} \sin ^{2} \phi_{0 i}+\sigma_{\perp, 0 i}^{2} \cos ^{2} \phi_{0 i}\end{array}\right)$,

where $\phi_{0 i}$ is the angle between $\vec{d}_{0 i}$ and our arbitrary Cartesian system measured counterclockwise from the $x$-axis, and we have introduced the notation that the uncertainty parallel to $\vec{d}_{i j}$ is $\sigma_{\|, i j}$ and the uncertainty orthogonal to $\vec{d}_{i j}$ is $\sigma_{\perp, i j}$ as calculated from Equation (1). Thus, we can identify the diagonal terms

$$
\begin{aligned}
\left\langle\Delta x_{0 i}^{2}\right\rangle & =\sigma_{\|, 0 i}^{2} \cos ^{2} \phi_{0 i}+\sigma_{\perp, 0 i}^{2} \sin ^{2} \phi_{0 i}, \\
\left\langle\Delta y_{0 i}^{2}\right\rangle & =\sigma_{\|, 0 i}^{2} \sin ^{2} \phi_{0 i}+\sigma_{\perp, 0 i}^{2} \cos ^{2} \phi_{0 i}
\end{aligned}
$$

and

$$
\left\langle\Delta x_{0 i} \Delta y_{0 i}\right\rangle=\left(\sigma_{\|, 0 i}^{2}-\sigma_{\perp, 0 i}^{2}\right) \cos \phi_{0 i} \sin \phi_{0 i} .
$$

For the off-diagonal terms $\left\langle\Delta x_{0 i} \Delta x_{0 j}\right\rangle$, we notice that (as used in Equation 14)

$$
\begin{aligned}
\left\langle\Delta x_{0 i} \Delta x_{0 j}\right\rangle= & \frac{1}{2}\left\langle\left\{\Delta x_{0 i}^{2}+\Delta x_{0 j}^{2}-\left[\Delta x_{0 i}-\Delta x_{0 j}\right]^{2}\right\}\right\rangle \\
= & \frac{1}{2}\left\{\left\langle\Delta x_{0 i}^{2}\right\rangle+\left\langle\Delta x_{0 j}^{2}\right\rangle-\left\langle\left[\Delta x_{0 i}-\Delta x_{0 j}\right]^{2}\right\rangle\right\} \\
= & \frac{1}{2}\left(\sigma_{\|, 0 i}^{2} \cos ^{2} \phi_{0 i}+\sigma_{\perp, 0 i}^{2} \sin ^{2} \phi_{0 i}+\sigma_{\|, 0 j}^{2} \cos ^{2} \phi_{0 j}\right. \\
& +\sigma_{\perp, 0 j}^{2} \sin ^{2} \phi_{0 j}-\sigma_{\|, i j}^{2} \cos ^{2} \phi_{i j} \\
& \left.-\sigma_{\perp, i j}^{2} \sin ^{2} \phi_{i j}\right)
\end{aligned}
$$

where, in the last step, we have used the fact that $x_{i j}=x_{0 i}-x_{0 j}$ and the relations in Equations (18) and (19). The quantities $\left\langle\Delta y_{0 i} \Delta y_{0 j}\right\rangle$ can be obtained by interchanging sine and cosine in Equation (20).

For the remaining off-diagonal terms $\left\langle\Delta x_{0 i} \Delta y_{0 j}\right\rangle$, we can use the fact that

$$
\begin{aligned}
\left\langle\Delta x_{0 i} \Delta y_{0 j}\right\rangle & =\left\langle\Delta x_{0 i}\left[\Delta y_{0 i}+\Delta y_{i j}\right]\right\rangle \\
& \left.=\left\langle\Delta x_{0 i} \Delta y_{0 i}\right\rangle+\left\langle\Delta x_{0 i} \Delta y_{i j}\right]\right\rangle
\end{aligned}
$$

$$
\begin{aligned}
= & \left\langle\Delta x_{0 i} \Delta y_{0 i}\right\rangle+\left\langle\left[\Delta x_{0 j}-\Delta x_{i j}\right] \Delta y_{i j}\right\rangle \\
= & \left\langle\Delta x_{0 i} \Delta y_{0 i}\right\rangle+\left\langle\Delta x_{0 j} \Delta y_{i j}\right\rangle-\left\langle\Delta x_{i j} \Delta y_{i j}\right\rangle \\
= & \left\langle\Delta x_{0 i} \Delta y_{0 i}\right\rangle+\left\langle\Delta x_{0 j}\left[\Delta y_{0 j}-\Delta y_{0 i}\right]\right\rangle-\left\langle\Delta x_{i j} \Delta y_{i j}\right\rangle \\
= & \left\langle\Delta x_{0 i} \Delta y_{0 i}\right\rangle+\left\langle\Delta x_{0 j} \Delta y_{0 j}\right\rangle-\left\langle\Delta x_{i j} \Delta y_{i j}\right\rangle \\
& -\left\langle\Delta x_{0 j} \Delta y_{0 i}\right\rangle .
\end{aligned}
$$

Rearranging gives

$$
\begin{gathered}
\left\langle\Delta x_{0 i} \Delta y_{0 j}\right\rangle+\left\langle\Delta x_{0 j} \Delta y_{0 i}\right\rangle=\left\langle\Delta x_{0 i} \Delta y_{0 i}\right\rangle \\
+\left\langle\Delta x_{0 j} \Delta y_{0 j}\right\rangle-\left\langle\Delta x_{i j} \Delta y_{i j}\right\rangle .
\end{gathered}
$$

All the terms on the right-hand side are known from Equation (19), and further investigation shows that the two terms on the left-hand side are equal. So, Equations (13), (14), (18)(20), and (22) contain all the information required to construct the full covariance matrix, $\boldsymbol{\Sigma}_{\mathbf{d}}$.

\subsection{The Optimal Weight Matrix}

The optimal choice of weights in Equation (3) are those that minimize the eigenvalues in Equation (11). For a $2 \times 2$ symmetric matrix, the sum of the eigenvalues is the trace of the matrix, so our problem becomes one of minimizing the trace of $\boldsymbol{\Sigma}_{\mathbf{p}}$ subject to the constraints in Equation (9). Specifically, we will use the method of Lagrange multipliers (Betts 1980) to find the optimal weights, $\mathbf{W}^{\prime}$, that minimize the quadratic equation

$$
\operatorname{Tr}\left(\boldsymbol{\Sigma}_{\mathbf{p}}\right)=\frac{1}{2} \mathbf{W}^{\prime \mathrm{T}} \mathbf{S} \mathbf{W}^{\prime}
$$

where

$$
\mathbf{S}=\left[\begin{array}{cc}
\boldsymbol{\Sigma}_{\mathbf{d}} & 0 \\
0 & \boldsymbol{\Sigma}_{\mathbf{d}}
\end{array}\right]
$$

is a $4 N \times 4 N$ matrix, and

$$
\begin{array}{r}
\mathbf{W}^{\prime}=\left[w_{x x, 01}, \ldots, w_{x x, 0 N}, w_{x y, 01}, \ldots, w_{x y, 0 N}\right. \\
\left.w_{y x, 01}, \ldots, w_{y x, 0 N}, w_{y y, 01}, \ldots, w_{y y, 0 N}\right]^{\mathrm{T}}
\end{array}
$$

is a vector of length $4 N$. Note that $\mathbf{W}^{\prime}$ has identical entries as $\mathbf{W}$ in Equation (8); it is just written as a single vector to cast the minimization problem into a single Equation (23). We want to find the extrema of Equation (23) subject to the linear constraints in Equation (9), which can be written as

$$
\mathbf{C W}^{\prime}=\mathbf{V}
$$

where we define the $4 \times 4 N$ symmetric matrix

$$
\mathbf{C}=\left[\begin{array}{cccccccccccc}
1 & \cdots & 1 & 0 & \cdots & 0 & 0 & \cdots & 0 & 0 & \cdots & 0 \\
& & & 1 & \cdots & 1 & 0 & \cdots & 0 & 0 & \cdots & 0 \\
& & & & & & 1 & \cdots & 1 & 0 & \cdots & 0 \\
\operatorname{sym} & & & & & & & & & 1 & \cdots & 1
\end{array}\right],
$$

and

$$
\mathbf{V}=\left[\begin{array}{l}
1 \\
0 \\
0 \\
1
\end{array}\right]
$$

In this framework, the optimal weights are those that solve the system of linear equations:

$$
\left[\begin{array}{cc}
\mathbf{S} & \mathbf{C}^{\mathrm{T}} \\
\mathbf{C} & \mathbf{0}
\end{array}\right]\left[\begin{array}{c}
\mathbf{W}^{\prime} \\
\lambda
\end{array}\right]=\left[\begin{array}{c}
\mathbf{0} \\
\mathbf{V}
\end{array}\right]
$$

Here, $\lambda$ are the Lagrange multipliers, which will not be used further. Equation (29) can be solved via a matrix inversion. 
Note that the general constraints on the weights we have written in Equations (9) and (26) have two somewhat unintuitive features. The first is that the $y$ measurements are sometimes used to compute the $x$ position and vice versa. The other property is that they allow for negative weights, meaning that in some cases, certain measurements will be subtracted in calculating the position of the astrometric target, $\vec{p}$. These two facts conspire to exploit the natural correlations inherent in the data. The flexible and possibly negative weights essentially allow the reference grid to be symmetrized, thereby using the known correlations to cancel noise so as to minimize the variance in $\vec{p}$.

\subsection{Numerical Simulations}

As indicated in the above analysis, the single-epoch uncertainty in the location, $\vec{p}$, of the target relative to the grid of reference stars is represented by the eigenvalues and eigenvectors of the $2 \times 2$ matrix $\Sigma_{\mathbf{p}}$ (Equation 11). This matrix itself depends on the distribution of reference stars, the precision of centering measurements, and the degree of noise correlation due to differential tilt through the matrix $\boldsymbol{\Sigma}_{\mathbf{d}}$. In this way, the intrinsic precision of the measured value of $\vec{p}$ depends on these three factors.

To ascertain the behavior of $\boldsymbol{\Sigma}_{\mathbf{p}}$ with the density of available reference stars, we performed a series of numerical simulations. In each simulation, $N(2 \leqslant N \leqslant 100)$ stars were randomly distributed throughout a $25^{\prime \prime} \times 25^{\prime \prime}$ FOV. We assumed that the target was a bright star in the middle of the field with a centering error of 0.5 mas and the reference stars were fainter, drawn from a Gaussian distribution with a mean centering error of 2 mas and a standard deviation of 1 mas (somewhat analogous to the situation for the guide star in M5; see Section 5). The full covariance matrix, $\boldsymbol{\Sigma}_{\mathbf{d}}$, was computed for each stellar configuration assuming these centering errors, the typical turbulence profile above Palomar Observatory, and a $1.4 \mathrm{~s}$ exposure time.

In the first simulation, $\boldsymbol{\Sigma}_{\mathbf{d}}$ was contracted as in Equation (11) using standard averaging for $\mathbf{W}\left(w_{x x, 0 i}=w_{y y, 0 i}=1 / N\right.$, $w_{x y, 0 i}=w_{y x, 0 i}=0$ ). For the second simulation, $\boldsymbol{\Sigma}_{\mathbf{d}}$ was contracted using the optimal $\mathbf{W}$ as calculated by using the prescription in Section 4.3. In each case, the geometric mean of the two eigenvalues of the resulting matrix, $\Sigma_{\mathbf{p}}$, was computed to form an estimate of the single-epoch measurement precision of $\vec{p}$. To average away random effects arising from the particular geometry of the random distribution of stars, each numerical simulation was repeated for 100 random distributions of stars for each value of $N$, and these were averaged to generate a mean value for the single-epoch measurement precision.

The resulting values for the single-epoch measurement precision of $\vec{p}$ are shown in Figure 2 as a function of the number of reference stars, along with the contributions of measurement noise and differential tilt jitter. In both simulations, the error due to measurement noise decreases as $N^{-0.3}$. However, in the limit of an infinite number of reference stars, this error asymptotes to the target star's measurement error. The rate at which the measurement noise decreases to this value depends on the distribution of reference star measurement errors.

The important distinction between the two simulations is the contribution of tilt jitter to astrometric performance. In the simulation utilizing standard averaging, there is very little gain with increased stellar density $\left(N^{-0.15}\right)$, and tilt jitter dominates the error budget. However, the optimal estimation algorithm rapidly $\left(N^{-0.7}\right)$ eliminates the contribution of differential tilt by taking advantage of the correlations inherent in $\boldsymbol{\Sigma}_{\mathbf{d}}$ and
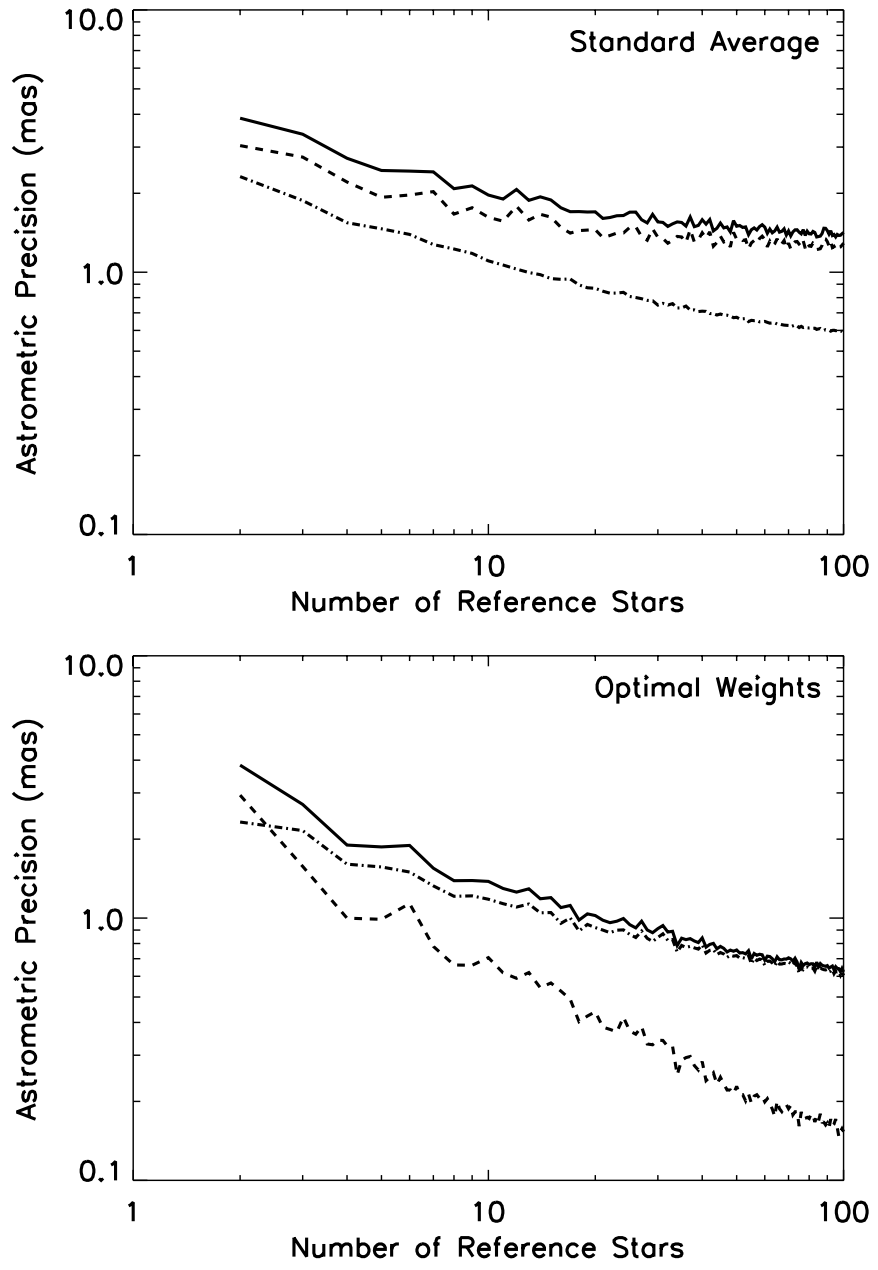

Figure 2. Top: simulated astrometric precision as a function of the number of reference stars using standard averaging (solid line). The total astrometric precision has contributions from the measurement noise (dash-dotted) and the differential atmospheric tilt jitter (dashed line). Here, the measurement noise was taken to be 0.5 mas for the $\mathrm{AO}$ guide star, and the reference stars were drawn from a normal distribution with a mean of 2 mas and a standard deviation of 1 mas. The tilt jitter is that expected in a $1.4 \mathrm{~s}$ exposure assuming the turbulence profile measured on the night of 2007 May 28 at Palomar Observatory (see Table 1). Bottom: simulated astrometric precision as a function of the number of reference stars using optimal weighting (lines as above). By using optimal weighting based on the covariance matrix, the effect of atmospheric noise is reduced to values less than measurement noise.

the flexibility to symmetrize the reference field through the choice of weights.

\section{ANALYSIS AND RESULTS}

In the analysis that follows, we will use the measurement model described in Section 4. For a given target star, we will calculate the differential offsets with respect to the grid stars to generate a value of $\mathbf{d}$ for each image at each epoch (Equation 6). We then use either these data or the theory in Section 4.2 to generate the full covariance matrix, $\boldsymbol{\Sigma}_{\mathbf{d}}$. From $\boldsymbol{\Sigma}_{\mathbf{d}}$, we use the prescription in Section 4.3 to calculate the optimal weights, W. These weights are used to combine the differential offsets to generate the target star's position, $\vec{p}$, in each image via Equation (7). The statistics of the positional measurements are then described by the covariance matrix, $\Sigma_{\mathbf{p}}$, from Equation (11). 


\subsection{Differential Tilt Jitter}

In order to test our expectation that tilt jitter dominates the astrometric error, we calculate the rms of the angular offsets for pairs of stars in the field (Figures 1 and 3). These results clearly show the characteristic signature of differential tilt. Namely, the rms separation along the axis connecting the two stars is larger than that of the perpendicular axis by a factor of $\approx \sqrt{3}$. However, the magnitude of the tilt jitter is smaller than the theoretical expectations, which suggests that some of the tilt jitter has been averaged away in the $1.4 \mathrm{~s}$ exposure time.

We have no direct measurement of the wind speed profile over the telescope to calculate the expected tilt jitter timescale. Instead, we fit the observed $\sigma_{i j}^{2}$ and angular offsets using the model in Equation (5) with $t=1.4 \mathrm{~s}$. The best-fit values are $\sigma_{\text {meas }, \mathrm{ij}} \approx 2$ mas and $t / \tau_{\mathrm{TJ}} \approx 7$. This implies that the characteristic timescale for tilt jitter is $\approx 0.2 \mathrm{~s}$, resulting in a wind crossing time of $25 \mathrm{~m} \mathrm{~s}^{-1}$. Turbulence at higher altitudes contributes most to the differential atmospheric tilt jitter, and this velocity is typical of wind speeds in the upper atmosphere (Greenwood 1977). It is also clear from the figure that a number of stars have measurement noise that is much less than 2 mas; thus, this number should only be taken as characteristic of the faint stars.

\subsection{Astrometric Precision}

The astrometric precision achieved in a single epoch is an important diagnostic of the measurement model. On a given night for a given star, we investigate the use of both the $\approx 500$ images and the theory in Section 4.2 to calculate $\boldsymbol{\Sigma}_{\mathbf{d}}$, leading to the optimal weights. We then apply this weight matrix to the measured offsets to compute the target's position in each image, resulting in a time series in each component of $\vec{p}$ for each epoch. The properties of each time series are best explored by computing its Allan deviation (also known as the square root of the two-sample variance). The Allan deviation is calculated by dividing a time series into chunks, averaging each segment, and computing the rms of the resulting, shorter time series. If the time series is dominated by random errors, its Allan deviation will decrease as $1 / \sqrt{t_{\mathrm{avg}}}$, where $t_{\mathrm{avg}}$ is the length of each chunk. It is also necessary to have sufficiently many segments so that an rms calculation is meaningful. Here, the longest timescale probed is $\approx 2-3$ minutes for each $10-15$ minute time series.

We compute the geometric mean of the Allan deviation in each dimension as a function of the averaging time for the $\mathrm{AO}$ guide star in Figure 4 after computing the covariance matrix from data. After $1.4 \mathrm{~s}$, the guide star's positional precision is $\approx 600 \mu$ as. The precision subsequently improves as $t^{-0.51 \pm 0.08}$ to $\approx 70 \mu$ as after 2 minutes, and has yet to hit a systematic floor. This suggests a precision of $\approx 30 \mu$ as for the full 10 15 minute data set, assuming that no systematic limit is reached in the interim.

This level of precision is not limited to the AO guide star; similar performance is obtained on other stars in the core of M5. In Figure 5, we show the astrometric precision obtained on 2007 May 29 after 2 minutes for all detected stars as a function of their $K_{s}$ magnitude. Precision below $100 \mu$ as is achieved on targets as faint as $K_{s} \approx 13 \mathrm{mag}$ using a narrowband filter and $1.4 \mathrm{~s}$ individual exposures. This demonstrates the substantial $\mathrm{S} / \mathrm{N}$ benefit afforded by $\mathrm{AO}$.

The astrometric precision shown in Figure 5 resulting from the theoretically-determined covariance matrix and optimal weights is $\approx 300 \mu$ as after 2 minutes for stars with $K_{s} \lesssim$
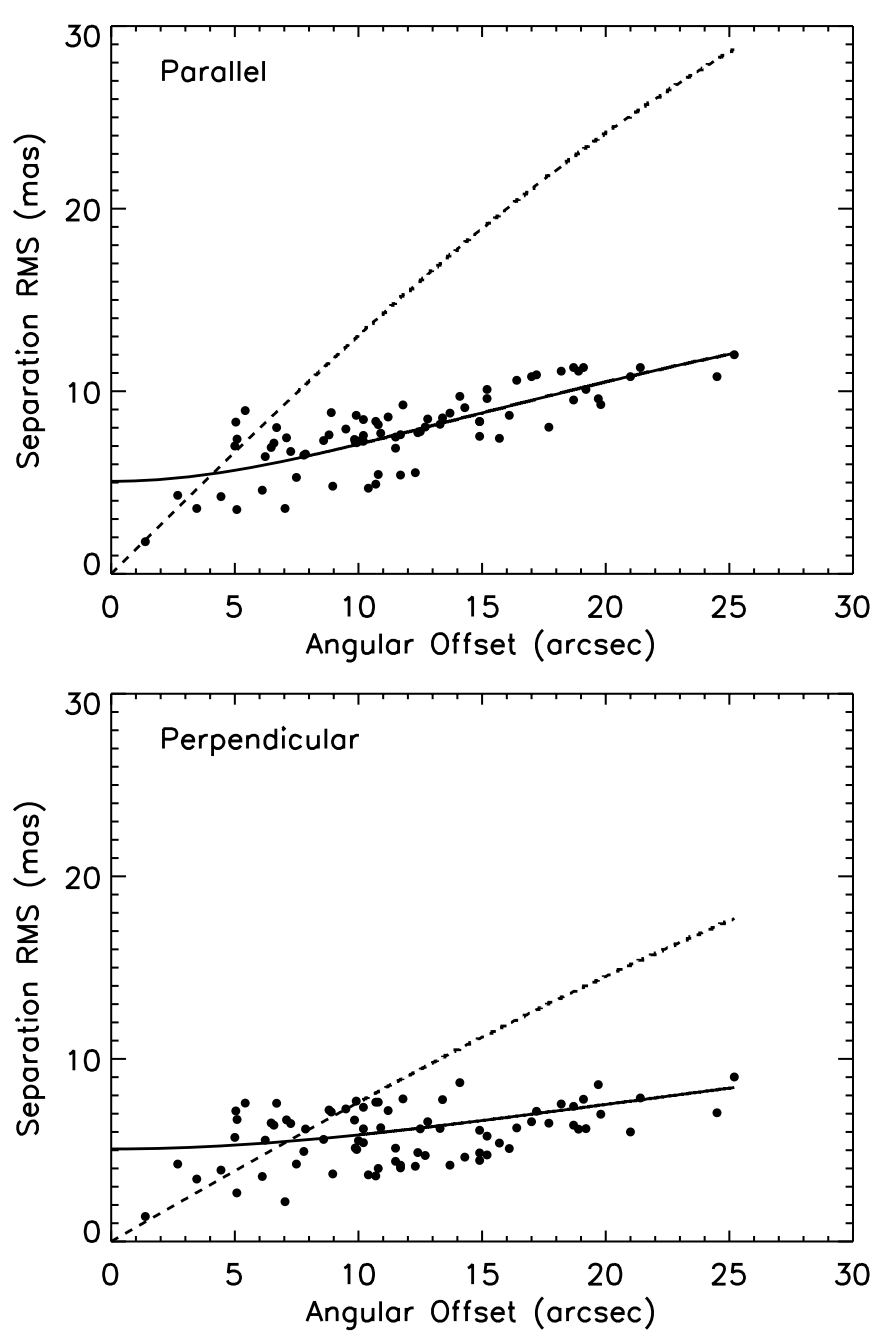

Figure 3. Top: rms deviation in the distance between pairs of stars in the direction parallel to their separation axis on 2007 May 28 (filled circles). The jitter predicted (assuming no measurement noise) from the measured turbulence profiles and Equation (1) (dashed line) is far larger than the measured jitter, indicating that some tilt jitter has been averaged away in $1.4 \mathrm{~s}$. The best-fit model (Equation 5), including averaged tilt jitter and measurement noise, indicates that the tilt timescale is $\approx 0.2 \mathrm{~s}$ (solid line). Bottom: as above, but in this case, the separations and predictions are for the direction perpendicular to the separation axis. The expected rms for the perpendicular direction is lower by the expected factor as seen in Equation (1). Note that not all pairs include the AO guide star.

13 mag. This level of precision is substantially better than the performance of simpler weighting schemes, but it is a factor of 2-4 worse than using the data to calculate the covariance matrix and weighting. There are several possible reasons for this reduction in precision. First, we have only used estimates of the measurement noise for each star used to calculate $\boldsymbol{\Sigma}_{\text {meas }}$. Second, the turbulence profile used to construct $\boldsymbol{\Sigma}_{\mathrm{TJ}}$ is estimated from the average $C_{n}^{2}(h)$ seen by the DIMM/MASS. This unit is located $300 \mathrm{~m}$ from the Hale telescope and uses Polaris to estimate the turbulence profile. As a consequence, there could be important differences between the measured atmospheric turbulence and that encountered by the light from M5. Finally, we have not attempted to capture the time variability of the turbulence, having used only the average values.

In Figure 6, we investigate the improvement of the AO guide star astrometry with the number of reference stars. We drew random subsets of the available grid stars, computed $\boldsymbol{\Sigma}_{\mathbf{d}}$ from the data, calculated the optimal weights, and showed the geometric 


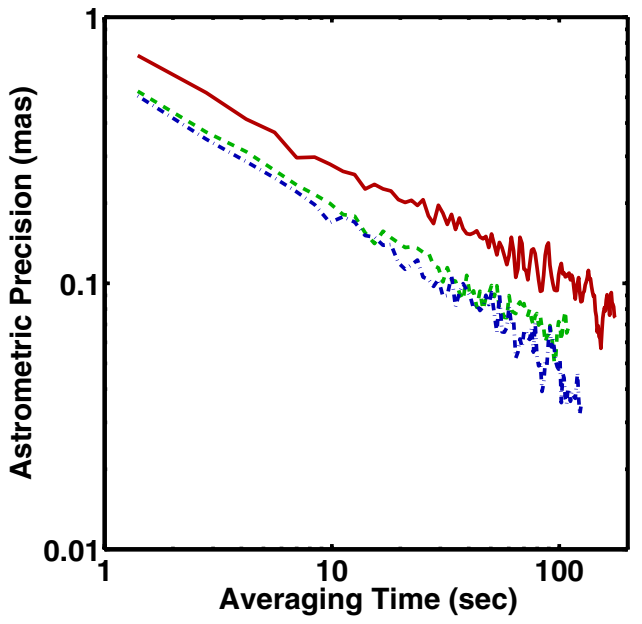

Figure 4. Allan deviation in the guide star position as a function of integration time for 2007 May 28 (solid curve), May 29 (dash-dotted), and July 22 (dashed). The astrometric precision scales as $t^{-0.51 \pm 0.08}$, and the covariance matrix and optimal weights were derived from data.

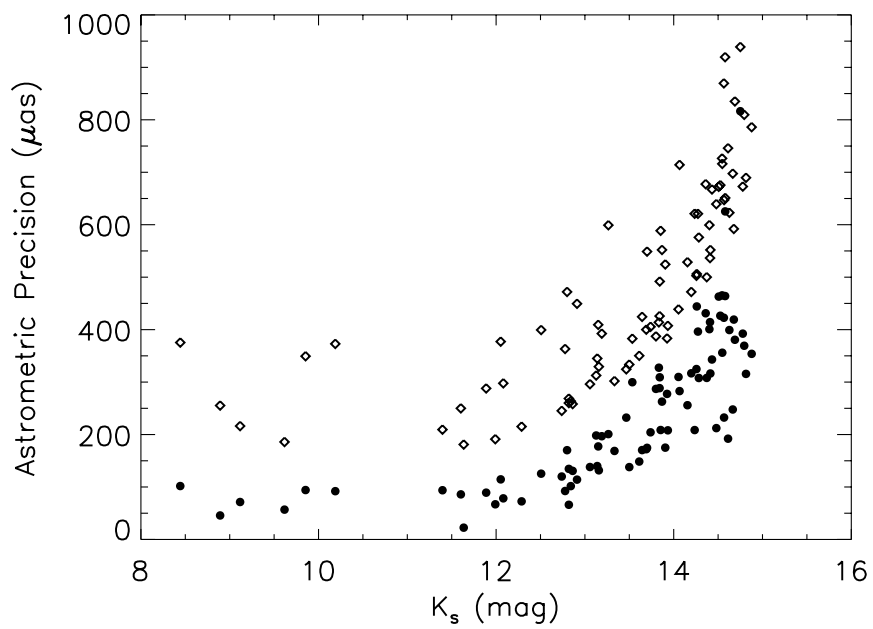

Figure 5. The astrometric precision (Allan deviation after 2 minutes) using the theoretical covariance matrix (open diamonds) and the covariance matrix from data (filled circles) as a function of $K_{s}$ magnitude for all 82 detected stars on 2007 May 29 . The precision in both cases is essentially constant for $K_{s} \lesssim 13$ mag. However, the astrometric precision for the theoretical $\Sigma_{\mathbf{d}}$ is a factor of 2-4 times larger than when calculated from data.

mean of the eigenvalues of $\boldsymbol{\Sigma}_{\mathbf{p}}$. To average over the geometry of a particular draw, we repeated this process ten times for each value of $N$ and averaged the results. We see that the precision rapidly decreases as $N^{-0.60 \pm 0.03}$. This is slightly faster than what our simulations predict for $1.4 \mathrm{~s}$ of integration time. However, as noted above, our simulations are meant to approximate M5, but do not capture the true distribution of stellar measurement errors (which are difficult to decouple from tilt jitter) or any evolution in atmospheric turbulence during the observation.

\subsection{Astrometric Accuracy}

The goal of astrometry is to measure the position of the target star over many epochs. Astrometrically interesting timescales range from hours to years. Clearly, the optical systems must be stable over these spans for astrometry with $\mathrm{AO}$ to be viable. There are several obstacles that could render the singleepoch precision obtained in Section 5.2 meaningless. For example, PHARO is mounted at the Cassegrain focus, which results in flexure of the instrument as the telescope tracks,

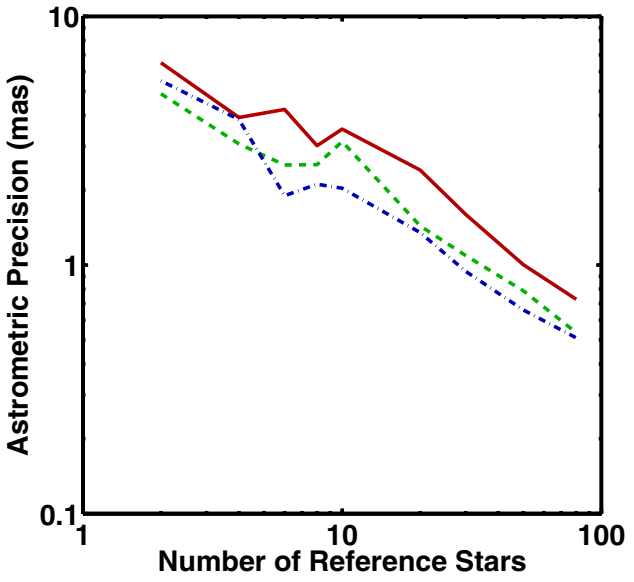

Figure 6. Allan deviation in $1.4 \mathrm{~s}$ for the $\mathrm{AO}$ guide star's position as a function of the number of reference stars on 2007 May 28 (solid curve), May 29 (dashdotted), and July 22 (dashed). The astrometric precision scales as $N^{-0.60 \pm 0.03}$.

and undergoes warming and cooling cycles between observing periods (typically twice per month) that could cause small changes in the powered optics. Either of these facts could alter the geometric distortion and make astrometric measurements unrepeatable. In order to probe the system stability, we have designed our experiment to be as consistent as possible, and it has spanned many removal and reinstallations of PHARO over 2 months.

In order to investigate the accuracy of the M5 measurements, we first measured and corrected the small rotational $(\lesssim 0.04)$ and plate scale $\left(\lesssim 10^{-5}\right)$ changes between the May 29 and July 22 data and the May 28 images. We also calculate the optimal weights for a given star on all three nights, and average them to create one weighting matrix to use for each epoch. This is not strictly optimal, since each night has different turbulence conditions for example, but it ensures that the scenario that $\vec{p} \rightarrow \vec{p}+\vec{\epsilon}$. In Figure 7, we see that the measured position of the AO guide star is accurate from epoch to epoch at $\approx 100 \mu$ as. The error ellipses are those estimated by continuing to extrapolate the precision found in Figure 4 by $1 / \sqrt{t}$ to the full 10 15 minute time series. This is an impressive level of accuracy, but unfortunately is a factor of 3 worse than our expectation. It suggests that there is some instability, likely in the distortion, over the 2 months that limit the astrometric accuracy.

The other stars in M5 show a similar level of astrometric accuracy (Figure 8) up to $K_{s} \approx 13 \mathrm{mag}$. This limit can be certainly pushed to be considerably fainter with increased integration time or a larger aperture. The achievement of such high levels of astrometric performance on faint targets, given the modest time investment, short integration time, and narrowband filters, illustrates the substantial $\mathrm{S} / \mathrm{N}$ gain and potential for astrometry enabled by $\mathrm{AO}$.

\section{DISCUSSION AND CONCLUSIONS}

Here we have presented a technique for performing high-precision grid astrometry using ground-based telescopes equipped with $\mathrm{AO}$ systems. With this technique, the effects of distortion and atmospheric dispersion that give rise to systematic errors are mitigated through the design of the experiment. Random errors arising from differential tilt jitter and measurement noise are minimized through the use of an optimal estimation scheme that accounts for the correlated noise statistics through the covariance matrix $\boldsymbol{\Sigma}_{\mathbf{d}}$. The experimental results obtained on 


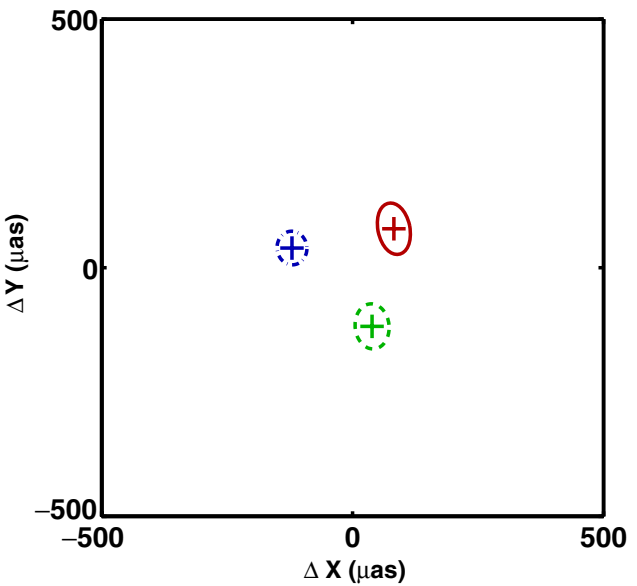

Figure 7. The position of the $\mathrm{AO}$ guide star in an arbitrary coordinate system on three dates: 2007 May 28 (solid), 2007 May 29 (dashed), and 2007 July 22 (dash-dotted; see Section 5.3). The error circles are inferred by averaging the covariance matrix measured from the data and extrapolating to the total $10-15$ minute integration time as $1 / \sqrt{t}$ (e.g., see Figure 4 ). The positions agree at the $\lesssim 100 \mu$ as level - a factor of $2-3$ larger than the expected dispersion. This discrepancy indicates that some systematic errors have occurred between epochs, most likely optical distortion.

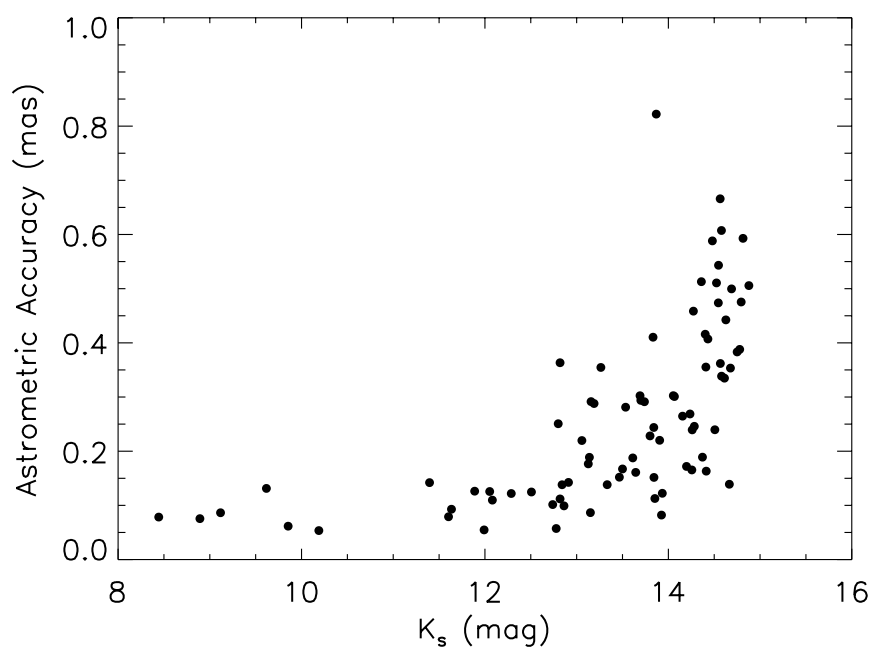

Figure 8. Astrometric accuracy (geometric mean of the rms in each coordinate over the three epochs) vs. $K_{s}$ magnitude. The level of accuracy is $\lesssim 100 \mu$ as, and is essentially unchanged for $K_{s} \lesssim 13 \mathrm{mag}$. However, this is a factor of 2-3 above our expectations from the achieved astrometric precision, suggesting a systematic limitation between epochs.

the Hale 200 inch telescope have demonstrated a single-epoch astrometric precision of $\lesssim 100 \mu$ as in 2 minutes and multiepoch astrometric accuracy at the same level. This level of precision is comparable to that afforded by ground-based interferometry, and is better than the precision obtained in seeing-limited programs on single apertures.

The simulation of astrometric precision afforded by the optimal weighting scheme, shown in Figure 2, illustrates that measurement noise is the dominant residual astrometric error on a $5 \mathrm{~m}$ telescope for stellar fields that contain more than a few reference stars. The scaling laws for differential tilt jitter $\left(D^{-7 / 6}\right)$ and measurement noise $\left(D^{-2}\right)$ indicate that on larger aperture telescopes, measurement noise will represent a smaller fraction of this residual error. This effect is illustrated in Figure 9, which shows the rms error between pairs of stars for a range of telescope apertures and angular separations.

The values in Figure 9 assume that tilt jitter is resolved by sufficiently short exposures. Longer exposure times will

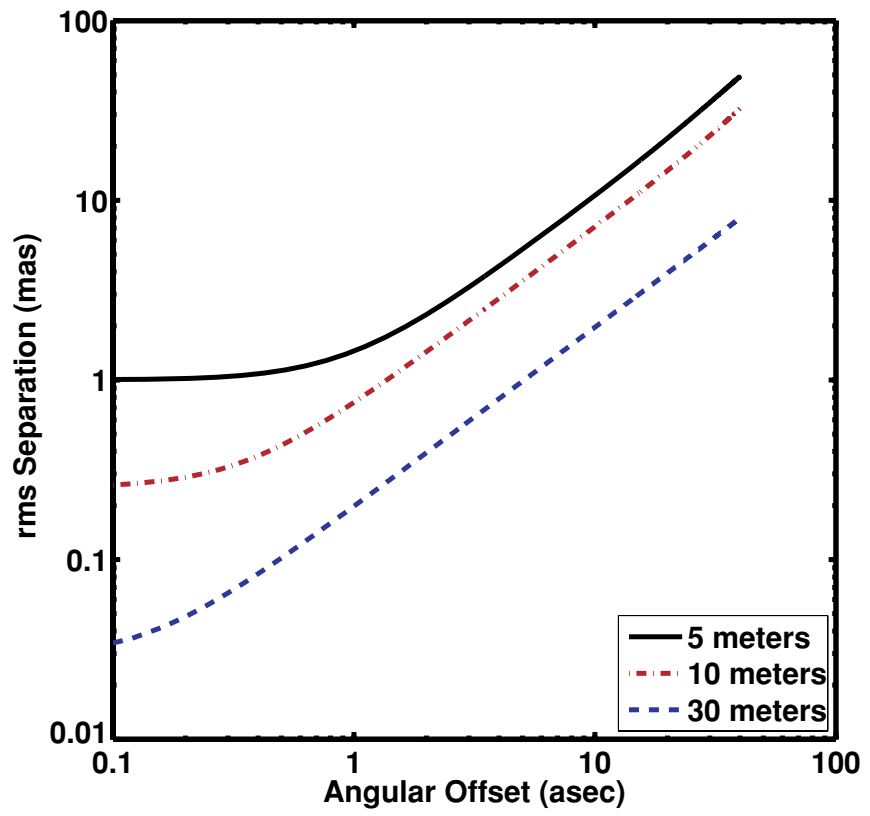

Figure 9. rms separation between a pair of stars vs. angular offset and aperture diameter. We have assumed the turbulence profile on 2007 May 28, a measurement error of $1 / \sqrt{2}$ mas for each star for a $5 \mathrm{~m}$ telescope, and included the geometric mean of each component of Equation (1). Relative to Palomar (solid curve), there are substantial astrometric gains to be made for larger $10 \mathrm{~m}$ (dash-dotted) and $30 \mathrm{~m}$ (dashed) telescopes due to the reduction of both measurement noise (the $y$-intercept; $\left.\propto D^{-2}\right)$ and tilt jitter $\left(\propto D^{-7 / 6}\right)$, respectively. Because measurement noise falls off more quickly with $D$, tilt jitter becomes the dominant source of astrometric error for large aperture telescopes.

certainly reduce the differential tilt jitter by $1 / \sqrt{t}$, but the measurement noise will also be decreased by this factor (for a given stellar brightness). The implication being that if tilt jitter dominates for short exposure times, it will continue to dominate longer exposures.

In situations where fewer images are available, either due to time constraints or longer exposure times per frame, it is difficult or impossible to effectively calculate the covariance matrix directly from the data. Our results show that independent measurements of the turbulence profile, for example from a DIMM/ MASS unit, are sufficient to calculate $\boldsymbol{\Sigma}_{\mathbf{d}}$, and result in astrometric precision within a factor of $2-4$ of the levels achieved using the data itself. Thus, the astrometric applications of turbulence sensors are twofold; they can be used to independently assess astrometric data quality and predict the AO PSF (Britton 2006).

The scaling laws presented throughout this paper indicate a substantially-improved astrometric performance on large aperture telescopes equipped with $\mathrm{AO}$. We have used the measured performance on M5 with the Hale Telescope combined with these scaling laws to predict the astrometric performance of a single conjugate $\mathrm{AO}$ system as a function of aperture diameter and number of reference stars. The relationship can be summarized, using the results of simulation and data analysis, as

$$
\begin{aligned}
\sigma_{\text {tot }}^{2}= & \sigma_{\text {meas }}^{2}+\sigma_{\mathrm{TJ}}^{2}=\left(\frac{1.4 \mathrm{sec}}{t}\right)\left\{\left[2 \operatorname{mas}\left(\frac{2}{N}\right)^{0.3}\left(\frac{5 \mathrm{~m}}{D}\right)^{2}\right]^{2}\right. \\
& \left.+\left[2 \operatorname{mas}\left(\frac{2}{N}\right)^{0.7}\left(\frac{5 \mathrm{~m}}{D}\right)^{7 / 6}\right]^{2}\right\}
\end{aligned}
$$




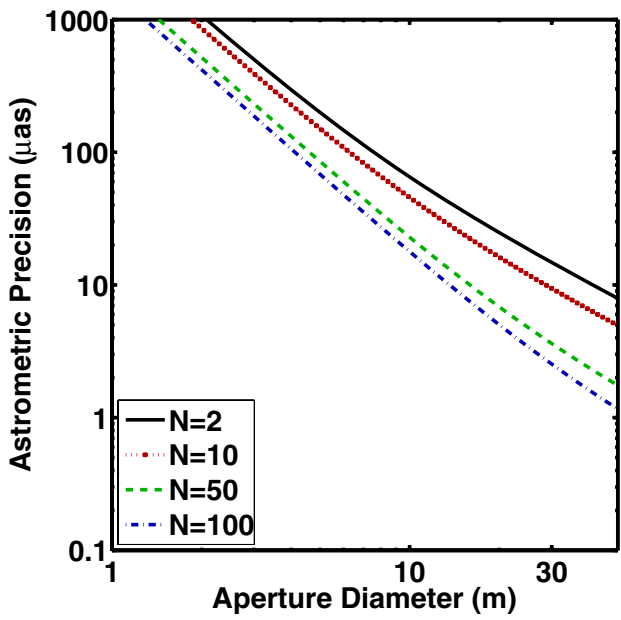

Figure 10. Astrometric precision as a function of aperture size and stellar density. We have used Equation (30) with the assumptions of the Palomar turbulence profile, a $25^{\prime \prime} \times 25^{\prime \prime} \mathrm{FOV}$, the M5 brightness distribution, and photon noise limit as described in Section 6. The astrometric precision demonstrates a very favorable scaling law with aperture diameter, and suggests that orders of magnitude improvement in precision may be available using large aperture, $\mathrm{AO}-$ equipped telescopes. In practice, the level of astrometric accuracy will depend on the extent to which current and future facilities can characterize and control systematic errors.

This equation assumes that measurement error is dominated by photon noise $\left(\propto D^{-2}\right)$, and the other dependences (FOV, stellar brightness distribution, turbulence profile) are identical to those for the M5 experiment.

Figure 10 shows the resulting estimates for astrometric precision as a function of aperture diameter and number of reference stars for a 2 minute exposure. These predictions demonstrate that limits to astrometric precision arising from random errors (dominated by tilt jitter) lie below $10 \mu$ as for $30 \mathrm{~m}$ telescopes. However, very careful characterization and control of systematic errors will be required to achieve this level of precision in an actual experiment. The extent to which systematic errors can be eliminated will distinguish the scientific goals that can be accomplished with ground-based facilities from those that require a space-based solution.

Facilities: Hale (PALAO/PHARO).

We thank N. Law, M. Ireland, D. Le Mignant, A. Kraus, M. van Kerkwijk, and A. Gould for useful discussions on astrometry. We also thank Palomar Observatory for providing support for the DIMM/MASS unit used in this study. This work has been supported by NASA, and by the National Science Foundation Science and Technology Center for Adaptive Optics, managed by the University of California at Santa Cruz under cooperative agreement no. AST-9876783.

\section{REFERENCES}

Anderson, J., Bedin, L. R., Piotto, G., Yadav, R. S., \& Bellini, A. 2006, A\&A, 454, 1029

Anderson, J., \& King, I. R. 2000, PASP, 112, 1360

Anderson, J., \& King, I. R. 2003a, PASP, 115, 113

Anderson, J., \& King, I. R. 2003b, AJ, 126, 772

Benedict, G. F., et al. 2003, AJ, 126, 2549

Betts, J. T. 1980, ACM Transactions on Mathematical Software, Vol. 6 (New York: ACM)

Boden, A. F., et al. 2007, ApJ, 670, 1214

Britton, M. C. 2006, PASP, 118, 885

Colavita, M. M., \& Wizinowich, P. L. 2003, in Proc. SPIE 4838, Interferometry for Optical Astronomy II, ed. W. A. Traub (Bellingham, WA: SPIE), 79

Diolaiti, E., Bendinelli, O., Bonaccini, D., Close, L. M., Currie, D. G., \& Parmeggiani, G. 2000, in Proc. SPIE 4007, Adaptive Optical Systems Technology, ed. P. L. Wizinowich (Bellingham, WA: SPIE), 879

Glindemann, A., et al. 2000, in Proc. SPIE 4006, Interferometry in Optical Astronomy, ed. P. J. Lena \& A. Quirrenbach (Bellingham, WA: SPIE), 2

Greenwood, D. P. 1977, J. Opt. Soc. Am. (1917-1983), 67, 390

Gubler, J., \& Tytler, D. 1998, PASP, 110, 738

Harris, W. E. 1996, AJ, 112, 1487

Hayward, T. L., Brandl, B., Pirger, B., Blacken, C., Gull, G. E., Schoenwald, J., \& Houck, J. R. 2001, PASP, 113, 105

Kornilov, V., Tokovinin, A., Shatsky, N., Voziakova, O., Potanin, S., \& Safonov, B. 2007, MNRAS, 382, 343

Lane, B. F., Colavita, M. M., Boden, A. F., \& Lawson, P. R. 2000, in Proc. SPIE 4006, Interferometry in Optical Astronomy, ed. P. J. Lena \& A. Quirrenbach (Bellingham, WA: SPIE), 452

Lazorenko, P. F. 2006, A\&A, 449, 1271

Lazorenko, P. F., Mayor, M., Dominik, M., Pepe, F., Segransan, D., \& Udry, S. 2007, A\&A, 471, 1057

Lindegren, L. 1978, in IAU Colloq. 48, Modern Astrometry, ed. F. V. Prochazka \& R. H. Tucker (Dordrecht: Kluwer), 197

Metchev, S. A. 2006, PhD thesis, California Institute of Technology

Monet, D. G., Dahn, C. C., Vrba, F. J., Harris, H. C., Pier, J. R., Luginbuhl, C. B., \& Ables, H. D. 1992, AJ, 103, 638

Muterspaugh, M. W., Lane, B. F., Konacki, M., Burke, B. F., Colavita, M. M. Kulkarni, S. R., \& Shao, M. 2006, A\&A, 446, 723

Perryman, M. A. C., et al. 2001, A\&A, 369, 339

Perryman, M. A. C., et al. 1997, A\&A, 323, L49

Pravdo, S. H., \& Shaklan, S. B. 1996, ApJ, 465, 264

Pravdo, S. H., Shaklan, S. B., Henry, T., \& Benedict, G. F. 2004, ApJ, 617,1323

Pryor, C., \& Meylan, G. 1993, in ASP Conf. Series 50, Structure and Dynamics of Globular Clusters, ed. S. G. Djorgovski, \& G. Meylan (San Francisco, CA: ASP), 357

Sandquist, E. L., \& Bolte, M. 2004, ApJ, 611, 323

Sasiela, R. J. 1994, Electromagnetic Wave Propagation in Turbulence. Evaluation and Application of Mellin Transforms (Springer Series on Wave Phenomena) (Berlin: Springer)

Stetson, P. B. 1987, PASP, 99, 191

Thomsen, M., Britton, M., \& Pickles, A. 2007, in AAS Meeting Abstracts 210 (Washington, DC: AAS), 117.01

Troy, M., et al. 2000, in Proc. SPIE 4007, Adaptive Optical Systems Technology, ed. P. L. Wizinowich (Bellingham, WA: SPIE), 31

Unwin, S. C., et al. 2008, PASP, 120, 863

Wizinowich, P. L., et al. 2006, PASP, 118, 297 